For reprint orders, please contact: reprints@futuremedicine.com

\title{
Analysis of melanoma recurrence following a negative sentinel lymph node biopsy
}

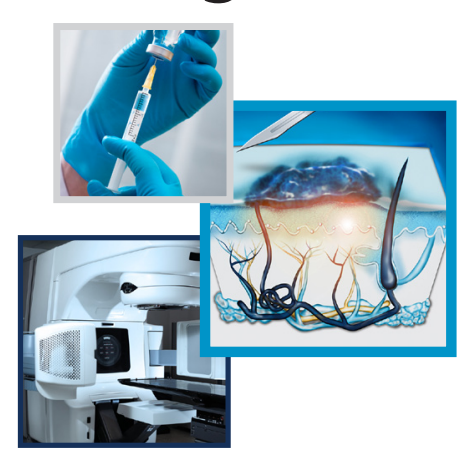

Maggie Hodges', Edward Jones', Teresa Jones', Nathan Pearlman', Csaba Gajdos', Nicole Kounalakis ${ }^{1} \&$ Martin McCarter,

\section{Practice points}

\section{Background}

- In 2008 the global burden of melanoma accounted for the loss of over 2 million disability-adjusted life years (DALYs) including approximately 1.9 million years of healthy life lost.

- In 2012,69,109 new cases of melanoma were diagnosed and 10,224 deaths were attributed to melanoma in the USA.

Current role of sentinel lymph node biopsy

- Since its introduction, sentinel lymph node biopsy (SLNBx) has been integrated into diagnostic algorithms for the evaluation of cutaneous melanoma.

- Current guidelines recommend wide local excision with SLNBx for stage $\mathrm{lb}$ and stage II melanomas that are $>0.1 \mathrm{~mm}$ in thickness and without clinical evidence of nodal disease.

- SLNBx determines the final staging and recommendations for systemic and surgical treatment options.

- The probability of developing distant or local disease recurrence following a negative sentinel lymph node (SLN) can range from 4 to $29 \%$.

- Little is reported regarding outcomes of patients with a 'false-negative' (FN) result.

Recurrence after negative SLNBX

- Median time to any melanoma recurrence after negative SLNBx was 9.3-35.4 months.

- Melanoma of the head and neck was observed to recur within the shortest time interval.

- Risk factors associated with recurrence included increased depth of the primary lesion, the presence of ulceration, advanced age at diagnosis and location of the primary lesion on the head and neck.

- Distant disease was the most common site of recurrence, representing 31.3-70.0\% of all patients who developed recurrent disease following a negative SLNBX.

\section{Definition of $\mathrm{FN}$ rate}

- A FN SLNBx is defined as recurrence of melanoma in the previously biopsied lymph node basin.

- The false omission rate or FN predictive value is defined as:

- (FN/initially negative SLNBX).

- The FN rate (FNR) is defined as: (FN/[true positive + FN]).

- False omission rate ranged from 0.9 to $8.9 \%$. 


\section{Practice points (cont.)}

\section{Definition of FN rate (cont.)}

- FNR ranged from 2.8 to $32.1 \%$.

- The highest FNR has been observed in patients undergoing SLNBx for head and neck melanoma.

- Five-year overall survival (OS) for patients following a positive SLNBx ranged from 32 to $69.7 \%$, and 5-year OS for patients who develop distant, local or regional disease after FN SLNBx ranged from 48.4 to $60 \%$.

- The majority of patients who develop disease recurrence after a negative SLNBX, do so following a true-negative SLNBx; however, no studies were found which evaluate outcomes or risk factors for recurrence in this population.

- The lack of data regarding survival of patients who develop local, regional or distant melanoma recurrence after truenegative SLNBX call for more in-depth evaluation of this patient population.

\section{Etiology of FN SLNBx}

- Possible etiologies of FN SLNBx include:

- Poor radiographic localization of the SLN;

- Failed pathologic evaluation of the sampled lymph node;

- Failure of surgical technique to identify the SLN.

\section{Melanoma biology \& recurrence}

- Melanoma which recurs after negative SLNBx may exhibit a different tumor biology.

- The development of distant metastases despite negative SLNBx may be due to:

- Local regression of primary melanoma lesions;

- Immunologic clearance of the melanoma in the regional lymph node basin;

- Direct hematogenous spread of the disease;

- The presence of melanoma leading to obstruction of lymphatic drainage.

\section{Follow-up of patients after negative SLNBX}

- Goal of improved risk stratification of melanoma patients at risk for recurrence based on individual patient's inherent biologic activity associated with a specific melanoma.

- The decreased OS of patients who develop recurrent melanoma following a negative SLNBx calls for increased efforts to:

- Mminimize FN SLNBx;

- Identify risk factors for recurrence;

- Define the roles of supplementary imaging (such as ultrasound) to aid the localizing of the true SLN;

- Improve understanding of individual melanoma tumor biology.

Little attention has been paid to the characteristics and outcomes of patients who experience distant, local or regional recurrence of melanoma following a negative sentinel lymph node biopsy. This article aims to review the published literature on the topic and presents some general summaries regarding this patient population. Patients who experience a disease recurrence following a negative sentinel lymph node biopsy have a worse overall survival compared with patients with a positive sentinel lymph node biopsy. The implications and possible explanations for these findings are discussed in order to both underscore the need for in-depth investigation of local, regional or distant melanoma recurrence among patients following a true negative sentinel lymph node biopsy, as well as increased efforts to minimize the rate of false negative sentinel lymph node biopsies. 
In 2008 the global burden of melanoma was estimated to account for the loss of over 2 million disability adjusted life years including approximately 1.9 million years of healthy life lost [1]. In 2012, 69,109 new cases of melanoma were diagnosed and 10,224 deaths were attributed to melanoma in the USA [2]. While the incidence of newly diagnosed melanoma has continued to increase, the mortality rate has been stable $[3,4]$. These trends likely reflect early detection coupled with preventative measures by public health organizations providing education to reduce ultraviolet light exposure via skin protection and avoidance of tanning beds [4].

\section{Current role of sentinel lymph node biopsy}

Sentinel lymph node biopsy (SLNBx) was first proposed in 1992 as a method of identifying high-risk patients who would most benefit from early, aggressive surgical and systemic therapies, as well as identifying the patients who could forego regional lymph node dissection, and thereby avoid the associated morbidity of a regional lymph node dissection [5]. Sentinel lymph node biopsy has since been hypothesized to prevent progression of regional disease [6,7]. Since its introduction, SLNBx has been rapidly integrated into diagnostic algorithms for the evaluation of cutaneous melanoma. Current National Comprehensive Cancer Network guidelines recommend wide local excision with SLNBx for stage Ib and stage II melanomas that are $>0.1 \mathrm{~mm}$ in thickness and without clinical evidence of nodal disease. This determines the final staging and appropriateness for additional systemic and surgical treatment options [8].

The sentinel lymph node (SLN) is defined as the first lymph node in the regional lymph node basin to receive drainage from the affected skin. During a SLNBx, the SLN is localized using two dyes which are both injected intradermally at the site of the primary lesion. The blue dye allows for intraoperative visualization of the SLN and results in an 80-85\% identification rate. The radioactive dye's migration to the SLN is traced with imaging (lymphoscintigraphy) and detected with an intraoperative gamma probe; this technique results in a $95 \%$ identification rate, whereas the technique combining use of both dyes demonstrates a $99 \%$ localization rate. The probability of developing distant or local disease recurrence following a negative SLN is largely dependent of the tumor depth and biology and can range from 4 to $29 \%[9,10]$.

While the overall outcomes of patients following positive and negative SLNBx have been well described in the literature, little is reported regarding patients with a 'false negative' (FN) result. Furthermore, the current literature lacks a clear definition for both a 'FN result' and for this patient population, which contributes to the challenge in reporting associated outcomes. Here, we review the current literature focusing upon this population and discuss future directions for investigation.

For this article, we performed a broad review of medical literature over the past 15 years using PubMed, Google Scholar, EBSCO Host with the following MeSH terms and keywords: 'melanoma', 'recurrence', 'sentinel lymph node biopsy', 'false negative' and 'outcome.' We included all studies that discussed local, regional or distant recurrence of melanoma after negative sentinel lymph node biopsy. When aggregating data, values and rates were most often averaged by weighting the study values by the inverse of the sample size of the source population. Survival data were aggregated using standard, random effects meta-analysis techniques [11], the results of which can be found in Figures 1 \& 2; however performance of this test was severely limited by the small number of studies which provided measures of data distribution (standard error or standard deviation), with which to perform the meta-analysis. Time to recurrence was most often presented as a median value, for which we were unable to perform a meta-analysis.

\section{Recurrence after negative SLNBx}

In the reviewed studies, the median time to any melanoma recurrence after negative SLNBx was 9.3 to 35.4 months following SLNBx; the average, median time to first recurrence is 21.8 months (Table 1). Melanoma of the head and neck was observed to recur within the shortest time interval (9.5-19 months following SLNBx) [10,12]. Risk factors associated with recurrence of melanoma following a negative SLNBx included increased depth of the primary lesion, the presence of ulceration, advanced age at diagnosis and location of the primary lesion on the head and neck [13,14]. Distant disease was the most common site of recurrence, representing $31.3-70.0 \%$ of all patients who developed recurrent disease following a negative SLNBx (mean: 46.2\%) (Table 2).

\section{KEYWORDS}

- false-negative rate

- melanoma • outcomes

- recurrence sentinel

lymph node • survival 


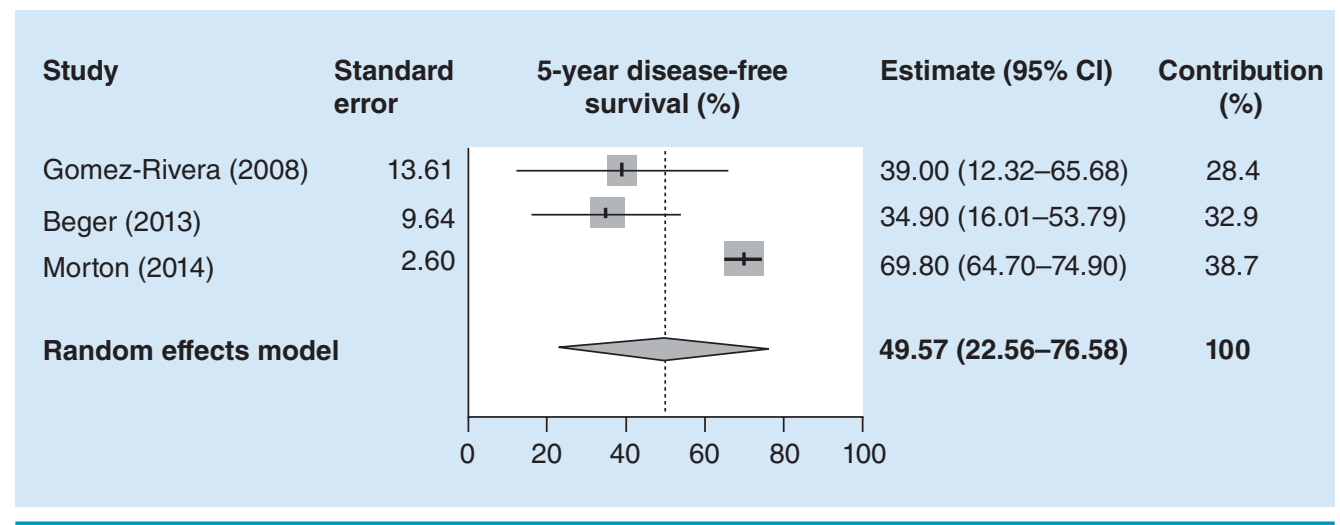

Figure 1. Results of meta-analysis evaluating 5-year disease free survival (\%) for patients following a positive sentinel lymph node biopsy.

\section{Definition of FN rate}

When considering recurrence of disease following a negative SLNBx, two populations of patients are often described: patients who develop recurrent disease after a true negative (TN) SLNBx, and patients who develop recurrent disease after a FN SLNBx. We define a FN SLNBx as recurrence of melanoma in the previously biopsied lymph node basin [13,15]. While the FN rate of SLNBx for cutaneous melanoma has been reported to broadly range from 1 to $32 \%$, attention should be paid to the manner of calculation of this value [16]. The FN rate (FNR) for a SLNBx has been previously calculated as the number of patients who develop disease recurrence in the locally draining lymph node basin (FN) divided by the number of patients with an initially negative SLNBx, or as the number of patients who recur in the locally draining lymph node basin divided by the total number of patients with a positive SLNBx (true positive + FN) [17]. While, the former definition (FN/initially negative SLNBx) provides an estimate of the false omission rate (FOR or FN predictive value) of a SLNBx for cutaneous melanoma [12], the more accurate method of calculating the FNR is represented by the latter definition (FN/true positive $+\mathrm{FN})$. This latter definition more appropriately estimates the proportion of patients who are initially incorrectly categorized as being free of disease in their sentinel lymph node following a negative SLNBx. When defined in this way, the FOR ranges from 0.9 to $8.9 \%$ (mean FOR 3.8\%, calculated using simple weighting by the inverse sample size of source study), while the FNR ranges from 2.8 to $32.1 \%$ (mean FNR 14.0\%, calculated using simple weighting by the inverse sample size of source study), as seen in Table 1. The highest FNRs have generally been observed in patients undergoing SLNBx for head and neck melanoma, with this association being attributed to the complex lymphatic drainage of the head and neck leading to increased technical failures due to difficulty identifying the SLN [18].

Patients who develop distant, local or regional disease following a negative SLNBx may have been incorrectly categorized by a FN SLNBx [13]. Direct comparison of the outcomes of patients who develop recurrent disease following negative SLNBx with patients whose SLNBx was positive reveals that reported 5 -year overall survival (OS) for patients following a positive SLNBx ranged from 32 to $69.7 \%$, and 5-year OS for patients who develop distant, local or regional disease after FN SLNBx ranged from 48.4 to $60 \%$ (mean $52 \%$, $95 \% \mathrm{CI}$ : 40.8-63.2\%). (Table 3 \& Figure 2). The mean 5-year OS for patients who develop distant, local or regional disease following FN SLNBx (calculated using simple weighting by the inverse of the source sample size) was $53.2 \%$ $(52 \%$ when calculated using meta-analysis techniques), compared with $61.8 \%$ for patients following a positive SLNBx. This expected decrease in 5-year OS could be the result of either more aggressive tumor pathology among patients with a TN or FN SLNBx, the presence of a therapeutic benefit afforded by SLNBx that patients with a FN SLNBx fail to receive (surgical technique alteration), or a delay in diagnosis resulting in lead time bias.

When the FN SLN is clearly defined, the majority of patients who develop disease after a negative SLNBx, do so following a TN SLNBx, meaning their disease develops outside of the 
regional lymphatic basin. However, none of the studies reviewed here specifically evaluate outcomes or risk factors for recurrence in this population. When focusing on the subset of patients who develop distant, local or regional recurrence after FN SLNBx, these patients also have both an expectedly poorer 5-year OS when compared with the total population of patients who undergo negative SLNBx. The 5-year OS of patients who develop distant, local or regional recurrence after FN SLNBx ranged from 48.4 to $60 \%$, compared with $70-92.7 \%$ [19] in the total population with negative SLNBx. While this discrepancy in survival is expected, the lack of data regarding survival of patients who develop local, regional or distant melanoma recurrence after TN SLNBx call for more in-depth evaluation of this patient population to identify specific risk factors and formulate guidelines for monitoring and evaluation of these patients following wide local excision and SLNBx.

Etiology of FN sentinel lymph node biopsy Possible etiologies of FN SLNBx include poor radiographic localization of the SLN, failed pathologic evaluation of the sampled lymph node and failure of surgical technique to identify the SLN. Poor reproducibility and operator error of cutaneous lymphoscintigraphy have been suggested as one etiology of FN SLNBx $[16,20]$. The presence of multiple sentinel lymph node basins has the potential to contribute to an increased risk of recurrence after negative SLNBx due to the complexity of the draining nodal systems; notably, the evaluation by Vidal et al. demonstrated the presence of multiple sentinel lymph node basins in up to $28 \%$ of patients [21]. In regions of the body where lymphatic drainage is known to be complex (head, neck), single-photon emission computerized tomography/computed tomography (SPECT/ CT) has been proposed as a method for better identifying lymph nodes with possible metastatic disease, with the use of SPECT/CT associated with a statistically significant disease-free survival benefit at 4 years $(93.9 \%$ survival in the SPECT/CT cohort vs $79.2 \%$ survival in the SLNBx cohort) [22]. Another source of possible FN SLNBx is surgical failure, wherein the operative surgeon fails to identify the SLN or samples non-sentinel lymph nodes in the targeted basin [7,23-24]. Furthermore, pathologic misclassification has been described as a potential source of FN SLNBx, with re-examination of sentinel lymph nodes demonstrated that 27 to $80 \%$ of patients who developed clinical progression of disease secondary to a FN SLNBx had histologic evidence of metastatic disease at the time of initial SLNBx $[7,16,25]$.

\section{Melanoma biology \& recurrence}

While every effort should be made to minimize the radiographic, histologic or surgical errors that lead to a FN SLNBx, it is also possible that melanoma which recurs after negative SLNBx exhibits a different tumor biology that is responsible for either the negative SLNBx, or the increased risk of recurrent disease. Current studies have identified phenotypic factors such as increased tumor thickness [7,1314,18,26-30], presence of ulceration [7,13-14,26-27,29$32]$, increased number of mitoses [26,29,31] and increased patient age [13-14,18,27] as risk factors for tumor recurrence. Attempts to further identify high-risk melanoma patients using molecular studies for melanoma markers such as in situ PCR and RT-PCR for MART1, MAGE-3, GalNAc-T and PAX3 mRNA have had some success, but suffer from the challenges of both broadly deploying complex technology as well

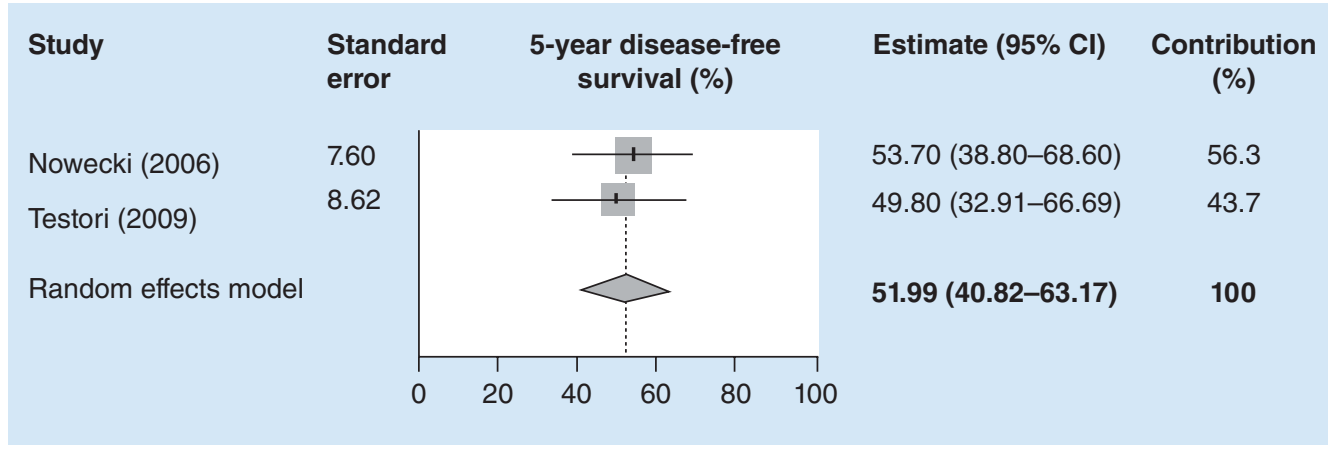

Figure 2. Results of meta-analysis evaluating 5-year overall survival (\%) for patients following a false-negative sentinel lymph node biopsy. 


\begin{tabular}{|c|c|c|c|c|c|c|c|c|c|c|c|c|}
\hline \multirow[t]{2}{*}{ Study, year } & \multirow[t]{2}{*}{$\mathrm{n}$} & \multirow{2}{*}{$\begin{array}{l}\text { Relative } \\
\text { weight } \\
(\%)\end{array}$} & \multirow{2}{*}{$\begin{array}{l}\text { TP } \\
\text { SLNBx } \\
\text { (n) }\end{array}$} & \multirow{2}{*}{$\begin{array}{l}(-) \\
\text { SLNBx } \\
\text { (n) }\end{array}$} & \multirow{2}{*}{$\begin{array}{l}\text { Median } \\
\text { follow-up } \\
\text { (months) }\end{array}$} & \multicolumn{2}{|c|}{$\begin{array}{l}\text { Any recurrence } \\
\text { after (-) SLNBx }\end{array}$} & \multicolumn{2}{|c|}{ FN SLNBx } & \multirow{2}{*}{$\begin{array}{l}\text { Time to } \\
\text { recurrence } \\
\text { (median, } \\
\text { months) }\end{array}$} & \multirow[t]{2}{*}{$\begin{array}{l}\text { FN rate } \\
(\%)\end{array}$} & \multirow{2}{*}{$\begin{array}{l}\text { Rate of any } \\
\text { recurrence } \\
\text { after TN } \\
\text { SLNBx (\%) }\end{array}$} \\
\hline & & & & & & $n$ & $\%$ & $n$ & $\%$ (FOR) & & & \\
\hline Yee, 2005 & 991 & 8.79 & 145 & 846 & 42.1 & 83 & 9.8 & 27 & 3.2 & 24.3 & 13.9 & 6.6 \\
\hline Zogakis, 2007 & 915 & 8.11 & & 773 & 36.7 & 69 & 8.9 & 13 & 1.7 & $23^{\ddagger}$ & 8.4 & 7.2 \\
\hline Dalal, 2007 & 1046 & 9.28 & 163 & 883 & 36 & 126 & 14.3 & 28 & 3.2 & 24 & 14.7 & 11.1 \\
\hline $\begin{array}{l}\text { Gomez-Rivera, } \\
2008^{+}\end{array}$ & 113 & 1.00 & 23 & 90 & 34 & 21 & 23.3 & 5 & 5.6 & & 17.9 & 17.8 \\
\hline Mattsson, 2008 & 361 & 3.20 & 73 & 288 & 12 & 39 & 13.5 & 12 & 4.2 & & 14.1 & 9.4 \\
\hline Chao, 2002 & 1183 & 10.49 & 233 & 950 & 16 & 58 & 6.1 & 11 & 1.2 & & 4.5 & 4.9 \\
\hline Clary, 2001 & 332 & 2.94 & 56 & 276 & 23 & 39 & 14.1 & 11 & 4.0 & 16.2 & 16.4 & 10.1 \\
\hline $\begin{array}{l}\text { Gershenwald, } \\
1998\end{array}$ & 295 & 2.62 & 52 & 243 & 35 & 27 & 11.1 & 14 & 5.8 & & 21.2 & 5.3 \\
\hline Carlson, 2008 & 1287 & 11.41 & 227 & 1060 & 44.3 & 133 & 12.5 & 35 & 3.3 & 23.9 & 13.4 & 9.2 \\
\hline Saltman, $2010^{\dagger}$ & 218 & 1.93 & 28 & 190 & 37 & 55 & 28.9 & 12 & 6.3 & 19 & 30.0 & 22.6 \\
\hline Savoia, 2012 & 688 & 6.10 & & 503 & 48 & 114 & 22.7 & 39 & 7.8 & 13.2 & 17.4 & 14.9 \\
\hline Rughani, 2010 & 694 & 6.15 & 162 & 532 & 46 & 53 & 10.0 & 16 & 3.0 & & 9.0 & 7.0 \\
\hline de Vries, 2011 & 429 & 3.80 & 119 & 310 & 64.8 & 69 & 22.3 & 17 & 5.5 & & 12.5 & 16.8 \\
\hline Miller, $2011^{\dagger}$ & 150 & 1.33 & 19 & 131 & 28.8 & 19 & 14.5 & 9 & 6.9 & & 32.1 & 7.6 \\
\hline Hinz, 2011 & 121 & 1.07 & 5 & 116 & 50.9 & 5 & 4.3 & 1 & 0.9 & $35.4^{9}$ & 16.7 & 3.4 \\
\hline $\begin{array}{l}\text { van den Broek, } \\
2013\end{array}$ & 305 & 2.70 & 54 & 251 & 50 & 24 & 9.6 & 6 & 2.4 & & 10.0 & 7.2 \\
\hline Fairbairn, $2012^{\S}$ & 66 & 0.59 & 22 & 44 & 36.8 & 10 & 22.7 & 2 & 4.5 & & 8.3 & 18.2 \\
\hline $\begin{array}{l}\text { Biver-Dalle, } \\
2012\end{array}$ & 197 & 1.75 & 44 & 153 & 39.5 & 20 & 13.1 & 10 & 6.5 & 17.4 & 18.5 & 6.5 \\
\hline Jones, 2013 & 619 & 5.49 & 104 & 520 & 61 & 83 & 16.0 & 21 & 4.0 & 23 & 17.5 & 11.9 \\
\hline Beger, 2013 & 201 & 1.78 & 33 & 168 & & 20 & 11.9 & 15 & 8.9 & 28.5 & 31.3 & 3.0 \\
\hline Sanchez, 2013 & 128 & 1.14 & 35 & 93 & 34 & 6 & 6.5 & 1 & 1.1 & & 2.8 & 5.4 \\
\hline Morton, 2014 & 765 & 6.78 & 122 & 643 & & & & 31 & & 21 & & \\
\hline Morton, $2014^{\S}$ & 173 & 1.53 & 57 & 116 & & & & 12 & & 9.3 & & \\
\hline Sum or range & 11277 & 100 & 1776 & 9179 & $12-64.8$ & 1073 & $4.3-28.9$ & 348 & $0.9-8.9$ & $9.3-35.4$ & $2.8-32.1$ & $3-22.6$ \\
\hline Average & 490 & & 85 & 400 & $38.9^{\#}$ & 76 & 12.8 & 21 & 3.8 & $21.8^{\#}$ & 14.0 & 9.2 \\
\hline \multicolumn{13}{|c|}{$\begin{array}{l}\text { TP SLNBx were considered the result of a positive initial sentinel lymph node biopsy. Negative SLNBx [(-) SLNBx] were considered the result of a negative initial sentinel lymph } \\
\text { node biopsy. } \\
\text { FN SLNBx were defined as the number of patients who developed recurrent melanoma in a nodal basin previously sampled during a negative initial SLNBx. } \\
\text { The FN rate was defined as the number of FN SLNBx divided by the sum of FN SLNBx and TP SLNBx: FN/(TP+FN)*100. } \\
\text { The FOR was calculated as the percent of initially negative SLNBx resulting in FN SLNBx: FN/(-) SLNBx*100. } \\
\text { The rate of recurrence after (-) SLNBx was calculated as the percent of patients with a negative initial SLNBx who develop recurrence of melanoma, regardless of } \\
\text { location: recurrence after (-) SLNBx/(-) SLNBx*100. }\end{array}$} \\
\hline \multicolumn{13}{|c|}{ 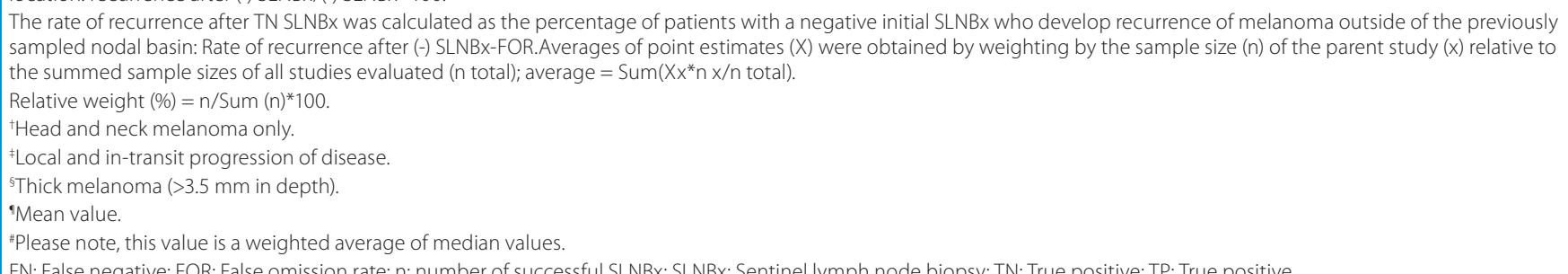 } \\
\hline
\end{tabular}

as relatively high false-positive results [33]. Similar to local regression of primary melanoma lesions, Yee et al. (2005), suggest the possibility that immunologic clearance of the melanoma in the regional lymph node basin prior to SLNBx may be responsible for the development of distant metastases despite negative SLNBx [26]. Among patients with head and neck melanoma, perhaps $55 \%$ of patients with melanoma-specific deaths developed metastatic disease without evidence of nodal involvement, suggesting a more direct hematogenous 
spread of the disease [7,12]. Similarly, metastatic melanoma leading to obstruction of lymphatic drainage has been proposed as a possible etiology of metastatic disease following a negative SLNBx, with case studies describing obstruction of lymphatic channels leading to failed lymphoscintigraphy, requiring ultrasound to diagnose the presence of metastatic nodal disease $[34,35]$. Management of distant or recurrent disease may be altered by clarifying the mechanism of melanoma metastasis.

\section{Follow-up of patients after negative sentinel lymph node biopsy}

Better risk stratification of melanoma patients at risk for recurrence awaits results from data analysis of genomic and protein sequencing that can assess the individual's inherent biologic activity associated with a specific melanoma. The decreased OS of patients who develop recurrent melanoma following a negative SLNBx calls for increased efforts to minimize FN SLNBx, identify risk factors for recurrence, define the roles of supplementary imaging (such as ultrasound) to aid the localizing of the true SLN and improve understanding of individual melanoma tumor biology. Follow-up of patients following a negative SLNBx can be challenging. National Comprehensive Cancer Network guidelines provide a range of options, from physical exam to routine imaging, depending on the estimated conditional probability of melanoma recurrence. For example, given that patients with primary melanoma of the head and neck develop recurrence of disease between 9.5 and 19 months after SLNBx, these patients should be monitored closely every 3-6 months after SLNBx. In the setting of a negative SLNBx, other factors that would suggest a greater risk of melanoma recurrence (and need for further

Table 2. Site of first recurrence after negative sentinel lymph node biopsy for cutaneous melanoma.

\begin{tabular}{|c|c|c|c|c|c|c|c|c|c|c|c|c|c|}
\hline \multirow[t]{3}{*}{ Study, year } & \multirow[t]{3}{*}{$\mathbf{n}$} & \multirow[t]{3}{*}{$\begin{array}{l}\text { Relative } \\
\text { weight } \\
(\%)\end{array}$} & \multirow[t]{3}{*}{$\begin{array}{l}(-) \\
\text { SLNBx } \\
\text { (n) }\end{array}$} & \multicolumn{2}{|c|}{$\begin{array}{l}\text { Recurrence } \\
\text { after (-) SLNBx } \\
\text { (n) }\end{array}$} & \multicolumn{8}{|c|}{ Site of first recurrence } \\
\hline & & & & \multirow[t]{2}{*}{$n$} & \multirow[t]{2}{*}{$\%$} & \multicolumn{2}{|c|}{ Distant } & \multicolumn{2}{|c|}{ Local } & \multicolumn{2}{|r|}{ Nodal } & \multicolumn{2}{|c|}{ In-transit } \\
\hline & & & & & & $n$ & $\%$ & $n$ & $\%$ & $n$ & $\%$ & $n$ & $\%$ \\
\hline Yee, 2005 & 991 & 10.07 & 846 & 83 & 9.8 & 45 & 54.2 & 6 & 7.2 & 22 & 26.5 & 10 & 12.0 \\
\hline Zogakis, 2007 & 915 & 9.30 & 773 & 69 & 8.9 & 37 & 53.6 & 9 & 13.0 & 13 & 18.8 & 14 & 20.3 \\
\hline $\begin{array}{l}\text { Gomez-Rivera, } \\
2008^{+}\end{array}$ & 113 & 1.15 & 90 & 21 & 23.3 & 10 & 47.6 & 6 & 28.6 & 7 & 33.3 & & \\
\hline Mattsson, 2008 & 361 & 3.67 & 288 & 39 & 13.5 & 14 & 35.9 & 11 & 28.2 & 12 & 30.8 & 2 & 5.1 \\
\hline Chao, 2002 & 1183 & 12.02 & 950 & 58 & 6.1 & 26 & 44.8 & 11 & 19.0 & 11 & 19.0 & 9 & 15.5 \\
\hline Clary, 2001 & 332 & 3.37 & 276 & 39 & 14.1 & 14 & 35.9 & 7 & 17.9 & 11 & 28.2 & 7 & 17.9 \\
\hline Rughani, 2010 & 694 & 7.05 & 532 & 53 & 10.0 & 19 & 35.8 & 11 & 20.8 & 17 & 32.1 & 6 & 11.3 \\
\hline de Vries, 2011 & 429 & 4.36 & 310 & 69 & 22.3 & 34 & 49.3 & 9 & 13.0 & 17 & 24.6 & 9 & 13.0 \\
\hline Hinz, 2011 & 121 & 1.23 & 116 & 5 & 4.3 & 3 & 60.0 & 1 & 20.0 & 1 & 20.0 & & \\
\hline $\begin{array}{l}\text { van den Broek, } \\
2013^{\ddagger}\end{array}$ & 305 & 3.10 & 251 & 24 & 9.6 & 9 & 37.5 & 6 & 25.0 & 6 & 25.0 & 2 & 8.3 \\
\hline Fairbairn, 2012 & 66 & 0.67 & 44 & 10 & 22.7 & 5 & 50.0 & 3 & 30.0 & 2 & 20.0 & & \\
\hline Biver-Dalle, 2012 & 197 & 2.00 & 153 & 20 & 13.1 & 14 & 70.0 & 8 & 40.0 & 10 & 50.0 & & \\
\hline Jones, 2013 & 619 & 6.29 & 520 & 83 & 16.0 & 26 & 31.3 & 19 & 22.9 & 21 & 25.3 & 12 & 14.5 \\
\hline Beger $2013^{\neq \S}$ & 201 & 2.04 & 168 & 20 & 11.9 & 7 & 35.0 & 7 & 35.0 & 15 & 75.0 & & \\
\hline Sum or Range & 9843 & 100 & 8006 & 993 & $4.3-22.7$ & 452 & $31.3-70.0$ & 191 & $6.3-40.0$ & 277 & $18.8-75.0$ & 105 & $5.1-29.6$ \\
\hline
\end{tabular}


Table 3. Five-year disease-free survival, overall survival, and melanoma-specific survival following false-negative and true-positive sentinel lymph node biopsy for cutaneous melanoma.

\begin{tabular}{|c|c|c|c|c|c|c|c|c|c|}
\hline \multirow[t]{2}{*}{ Study, year } & \multirow[t]{2}{*}{$\mathbf{n}$} & \multirow{2}{*}{$\begin{array}{l}\text { Relative } \\
\text { weight (\%) }\end{array}$} & \multirow{2}{*}{$\begin{array}{l}\text { TP SLNBx } \\
\text { (n) }\end{array}$} & \multirow{2}{*}{$\begin{array}{l}\text { (-) SLNBx } \\
\text { (n) }\end{array}$} & \multirow{2}{*}{$\begin{array}{l}\text { Median } \\
\text { follow-up } \\
\text { (months) }\end{array}$} & \multicolumn{2}{|c|}{ TP SLNBx (5 year, \%) } & \multicolumn{2}{|c|}{ FN SLNBx (5 year, \%) } \\
\hline & & & & & & DFS & os & MSS & OS \\
\hline Yee, 2005 & 991 & 8.14 & 145 & 846 & 42.1 & 43.5 & 56.0 & & \\
\hline Dalal, 2007 & 1046 & 8.59 & 163 & 883 & 36.0 & 72.0 & & & \\
\hline Caraco, $2007^{\dagger}$ & 801 & 6.57 & 151 & 650 & 42.8 & 52.0 & 66.3 & & 48.4 \\
\hline Gajdos, $2009^{\S}$ & 227 & 1.86 & 107 & 120 & 43.0 & 47.8 & 47.0 & & \\
\hline Testori, 2009 & 1313 & 10.78 & 229 & 1084 & 54.2 & & 67.7 & & 49.8 \\
\hline Scoggins, 2010 & 2451 & 20.12 & 486 & 1965 & 61.0 & 69.1 & 62.3 & & 52.8 \\
\hline Kelly, 2012 & 57 & 0.47 & 18 & 39 & & 11.0 & 32.0 & & \\
\hline Van den Broek, 2013 & 305 & 2.50 & 54 & 251 & 50.0 & 41.0 & & & \\
\hline Jones, 2013 & 619 & 5.08 & 99 & 520 & 61.0 & & 63.0 & & \\
\hline Beger, 2013 & 201 & 1.65 & 33 & 168 & & 34.9 & & & \\
\hline Morton, $2014^{\dagger}$ & 765 & 6.27 & 122 & 63 & & 69.8 & & 45.2 & \\
\hline Morton, $2014^{\dagger, \S}$ & 173 & 1.42 & 57 & 116 & & 60.8 & & 19.4 & \\
\hline Sum or Range & 12184 & 100 & 2305 & 9879 & $34.0-96.0$ & $11-69.8$ & $32-69.7$ & $19.4-62.5$ & $48.4-60.0$ \\
\hline Average & 677 & & 128 & 549 & 48.3 & 59.0 & 61.8 & 46.6 & 53.2 \\
\hline \multicolumn{10}{|c|}{$\begin{array}{l}\text { False-negative cases of melanoma only. } \\
\text { fHead and neck melanoma only. } \\
\text { SThick melanoma (>3.5 mm in depth). } \\
\text { DFS: Disease-free survival; FN: False negative; MSS: Melanoma-specific survival; n: Number of successful SLNBx; OS: Overall survival: SLNBx: Sentinel lymph node biopsy; TP: True } \\
\text { positive. }\end{array}$} \\
\hline
\end{tabular}

monitoring) include increased depth, the presence of ulceration and increased number of mitoses of the primary melanoma lesion, as well as increased age of the patient at the time of SLNBx.

\section{Conclusion}

Of patients undergoing a SLNBx, approximatley $13 \%$ experience a recurrence at an average of 21 months following the procedure. Risk factors associated with recurrence include increasing depth of the primary melanoma, ulceration of the primary, older age at diagnosis and primary location on the head and neck. A distant site of recurrence was more common than local or regional recurrences. Overall survival at 5 years for patients experiencing a recurrence after a negative SLNBx is approximately $52 \%$.

\section{Future perspective}

As research progresses forward, we encourage future efforts to be directed at investigating outcomes associated with recurrence following true-negative SLNBx. Furthermore, we suggest standardization in the presentation of outcomes data; including the definition of FN sentinel lymph node biopsy, calculation of FNRs and false omission rates, and presentation of data in a format allowing aggregation using meta-analysis techniques (including measures of central tendency and data spread). As time progresses, our hope and expectation is that ongoing basic science research will further define molecular markers able to predict risk of recurrence and enable the development of tailored, individualized chemotherapeutic regimens for our patients.

Financial \& competing interests disclosure The authors have no relevant affiliations or financial involvement with any organization or entity with a financial interest in or financial conflict with the subject matter or materials discussed in the manuscript. This includes employment, consultancies, honoraria, stock ownership or options, expert testimony, grants or patents received or pending, or royalties.

No writing assistance was utilized in the production of this manuscript. 


\section{References}

Papers of special note have been highlighted as:

- of interest

1 Soerjomataram I, Lortet-Tieulent J, Parkin DM et al. Global burden of cancer in 2008: a systematic analysis of disability-adjusted life-years in 12 world regions. Lancet 380 (9856), 1840-1850 (2012).

2 International Agency for Research on Cancer. GLOBOCAN 2012: estimated cancer incidence, mortality and prevalence worldwide in 2012. World Health Organization (2014).

http://globocan.iarc.fr

3 Purdue MP, Freeman LE, Anderson WF, Tucker MA. Recent trends in incidence of cutaneous melanoma among US Caucasian young adults. J. Invest. Dermatol. 128(12), 2905-2908 (2008).

4 Watson M, Johnson CJ, Chen VW et al. Melanoma surveillance in the United States: overview of methods. J. Am. Acad. Dermatol. 65 (5 Suppl. 1), S6-S16 (2011).

5 Morton DL, Wen DR, Wong JH et al. Technical details of intraoperative lymphatic mapping for early stage melanoma. Arch. Surg. 127(4), 392-399 (1992).

6 Boland GM, Gershenwald JE. Sentinel lymph node biopsy in melanoma. Cancer J. 18(2), 185-191 (2012).

7 Gershenwald JE, Colome MI, Lee JE et al. Patterns of recurrence following a negative sentinel lymph node biopsy in 243 patients with stage I or II melanoma. J. Clin. Oncol. 16(6), 2253-2260 (1998).

8 Coit DG, Thompson JA, Andtbacka R et al. NCCN Guidelines: melanoma (version 4.2014). J. Natl Compr. Canc. Netw. 12(5), 621-629 (2014).

- Guidelines for evaluation and treatment of patients with cutaneous melanoma.

9 Hinz T, Ahmadzadehfar H, Wierzbicki A et al. Prognostic value of sentinel lymph node biopsy in 121 low-risk melanomas (tumour thickness $<1.00 \mathrm{~mm}$ ) on the basis of a long-term follow-up. Eur. J. Nucl. Med. Mol. Imaging 39(4), 581-588 (2012).

10 Saltman BE, Ganly I, Patel SG et al. Prognostic implication of sentinel lymph node biopsy in cutaneous head and neck melanoma. Head Neck 32(12), 1686-1692 (2010).

11 Borenstein M, Hedges LV, Higgins JPT, Rothstein HR. A basic introduction to fixed-effect and random-effects models for meta-analysis. Res. Synth. Methods 1(2), 97-111 (2010).
12 Parrett BM, Kashani-Sabet M, Singer MI et al. Long-term prognosis and significance of the sentinel lymph node in head and neck melanoma. Otolaryngol. Head Neck Surg. 147(4), 699-706 (2012).

13 Jones EL, Jones TS, Pearlman NW et al. Long-term follow-up and survival of patients following a recurrence of melanoma after a negative sentinel lymph node biopsy result. JAMA Surg. 148(5), 456-461 (2013).

- Initial identification that patients who develop recurrence after true-negative sentinel lymph node biopsy are an understudied population.

14 Savoia P, Fava P, Caliendo V et al. Disease progression in melanoma patients with negative sentinel lymph node: does false-negative specimens entirely account for this phenomenon? J. Eur. Acad. Dermatol. Venereol. 26(2), 242-248 (2012).

15 Scoggins CR, Martin RC, Ross MI et al. Factors associated with false-negative sentinel lymph node biopsy in melanoma patients. Ann. Surg. Oncol. 17(3), 709-717 (2010).

- Large case series evaluating risk factors for recurrence after false-negative sentinel lymph node biopsy.

16 Miller MW, Vetto JT, Monroe MM, Weerasinghe R, Andersen PE, Gross ND. False-negative sentinel lymph node biopsy in head and neck melanoma. Otolaryngol. Head Neck Surg 145(4), 606-611 (2011).

17 Nieweg OE, Veenstra HJ. False-negative sentinel node biopsy in melanoma. J. Surg. Oncol. 104(7), 709-710 (2011).

18 Carlson GW, Page AJ, Cohen C et al. Regional recurrence after negative sentinel lymph node biopsy for melanoma. Ann. Surg. 248(3), 378-386 (2008).

- Large case series of melanoma recurrence after negative sentinel lymph node biopsy.

19 Morton DL, Thompson JF, Cochran AJ et al. Final trial report of sentinel-node biopsy versus nodal observation in melanoma. N. Engl. J. Med. 370 (7), 599-609 (2014).

- Phase III trial evaluating sentinel lymph node biopsy versus nodal observation in cutaneous melanoma.

20 Sondak VK, Zager JS. Who is to blame for false-negative sentinel node biopsies in melanoma? Ann. Surg. Oncol. 17(3), 670-673 (2010).

21 Mattsson J, Bergkvist L, Abdiu A et al. Sentinel node biopsy in malignant melanoma: Swedish experiences 1997-2005. Acta Oncol. 47(8), 1519-1525 (2008).
22 Stoffels I, Boy C, Poppel T et al. Association between sentinel lymph node excision with or without preoperative SPECT/CT and metastatic node detection and disease-free survival in melanoma. JAMA 308(10), 1007-1014 (2012).

23 Amersi F, Morton DL. The role of sentinel lymph node biopsy in the management of melanoma. Adv. Surg. 41, 241-256 (2007).

24 Lee NC, Spillane AJ, Pang TC, Haydu LE, Uren RF. Incomplete sentinel node biopsy is not clearly related to survival or regional recurrence in cutaneous melanoma patients. Ann. Surg. Oncol. 19(1), 280-286 (2012).

25 Itakura E, Huang RR, Wen DR, Cochran AJ. "Stealth" melanoma cells in histologynegative sentinel lymph nodes. Am. J. Surg. Pathol. 35(11), 1657-1665 (2011).

26 Yee VS, Thompson JF, McKinnon JG et al. Outcome in 846 cutaneous melanoma patients from a single center after a negative sentinel node biopsy. Ann. Surg. Oncol. 12(6), 429-439 (2005).

27 Zogakis TG, Essner R, Wang HJ, Foshag LJ, Morton DL. Natural history of melanoma in 773 patients with tumor-negative sentinel lymph nodes. Ann. Surg. Oncol. 14(5), 1604-1611 (2007).

28 Clary BM, Brady MS, Lewis JJ, Coit DG. Sentinel lymph node biopsy in the management of patients with primary cutaneous melanoma: review of a large single-institutional experience with an emphasis on recurrence. Ann. Surg. 233(2), 250-258 (2001)

29 Gajdos C, Griffith KA, Wong SL et al. Is there a benefit to sentinel lymph node biopsy in patients with T4 melanoma? Cancer 115(24), 5752-5760 (2009).

30 Biver-Dalle C, Puzenat E, Puyraveau M et al. Sentinel lymph node biopsy in melanoma: our 8 -year clinical experience in a single French institute (2002-2009). BMC Dermatol. 12, 21 (2012)

31 Rughani MG, Swan MC, Adams TS et al. Sentinel lymph node biopsy in melanoma: the Oxford ten year clinical experience. J. Plast. Reconstr. Aesthet. Surg. 64(10), 1284-1290 (2011).

32 Nowecki ZI, Rutkowski P, NasierowskaGuttmejer A, Ruka W. Survival analysis and clinicopathological factors associated with false-negative sentinel lymph node biopsy findings in patients with cutaneous melanoma. Ann. Surg. Oncol. 13(12), 1655-1663 (2006).

33 Ross MI, Gershenwald JE. Sentinel lymph node biopsy for melanoma: a critical update 
SYSTEMATIC REVIEW Hodges, Jones, Jones et al.

for dermatologists after two decades of experience. Clin. Dermatol. 31(3), 298-310 (2013).

- Broad review addressing current state and future direction of melanoma research.

34 Lam TK, Uren RF, Scolyer RA, Quinn MJ, Shannon KF, Thompson JF. False-negative sentinel node biopsy because of obstruction of lymphatics by metastatic melanoma: the value of ultrasound in conjunction with preoperative lymphoscintigraphy. Melanoma Res. 19(2), 94-99 (2009).

35 Iwata H, Kamiya H, Kitajima Y. Falsenegative sentinel lymph node biopsy resulting from obstruction of lymphatic basin by nodal metastasis: a case report of malignant melanoma. J. Dermatol. 38(11), 1121-1124 (2011).

- Valuable discussion regarding alternative etiologies of false-negative lymph node biopsy. 